\title{
Modeling and Simulation of Linear Two - DOF Vehicle Handling Stability
}

\author{
Pei-Cheng SHI ${ }^{a}$, Qi ZHAO and Shan-Shan PENG \\ Anhui Polytechnic University, Anhui Engineering Technology Research Center of Automotive New Technique, \\ 241000 Wuhu, China
}

\begin{abstract}
Based on the theory of vehicle steering dynamics, a model of linear two-DOF vehicle handling stability was established in MATLAB / Simulink environment. Simulation analysis of state variable response such as vehicle yaw velocity and side slip angle was made under different vehicle speed conditions for step-input of front-wheel turning angle. The response curves of the state variables in the time domain are obtained under three vehicle speeds. The results show that the response curve of simulation is in good agreement with the actual situation, which not only verifies correctness of the established vehicle handling stability model, but also provides theoretical basis for subsequent design of handling stability control system.
\end{abstract}

\section{Introduction}

Vehicle handling stability is one of the important characteristics of vehicle, which directly affects driving convenience, vehicle dynamics and driver and passenger safety[1]. Vehicle handling stability includes two parts of controllabitity and stability. Controllabitity refers to the extent to which the vehicle is able to respond to driver's steering commands when the driver does not feel tired or nervous. Stability refers to the ability of the vehicle to maintain stable driving when encountering external disturbances (road disturbance or gust disturbance). The two are interrelated, affecting vehicle handling stability together. With the continuous improvement of vehicle speed, research on vehicle handling stability has attracted increasingly wider attention $[2,3]$.

In the study of vehicle handling stability, yaw velocity and slip angle are generally considered as the optimal state variables to describe vehicle dynamics stability ${ }^{[4]}$, which reflect vehicle stability from different sides. In this paper, a linear two-DOF vehicle handling stability model and analog simulation were established by MATLAB / Simulink. The vehicle yaw velocity and side slip angle changes were compared and analyzed under different vehicle speeds at front wheel turning angle step input. The results have certain engineering application value.

\section{Vehicle handling stability modeling}

In order to simulate the basic characteristics of vehicle handling stability, the vehicle model is reduced to an ideal linear two-DOF vehicle model. Two-DOF refers to two state variables: yaw velocity and

\footnotetext{
${ }^{a}$ Corresponding author: 18355311303@163.com
} 
side slip angle. The model assumes that vehicle only makes plane movement parallel to the ground. That is, vehicle displacement along $\mathrm{z}$-axis, pitch angle around the $\mathrm{y}$-axis and roll angle around $\mathrm{x}$-axis are zero. Also, the origin of the vehicle coordinate system coincides with center of mass of vehicle. The simplified vehicle model is shown in Figure. 1.

The modeling is also based on the assumption that the driving force is not large and effect of ground tangential force on laterodeviation characteristic is not taken into account, effect of the air resistance is not considered, and tire characteristic changes of the left and right wheel tires due to load variation and aligning torque of the wheel are ignored.

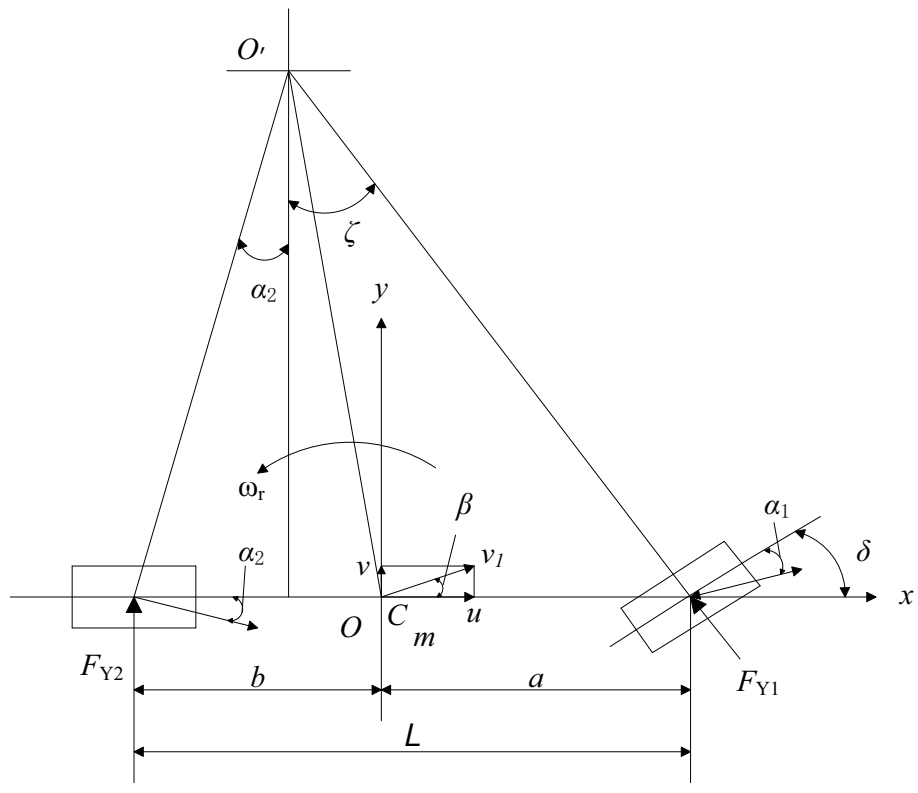

Figure 1. Two-DOF vehicle model.

In the Figure: $a, b$ are respectively distance between the front and rear axles to the center of mass of the vehicle; $L$ is vehicle wheelbase; $\omega_{r}$ is vehicle yaw velocity; $\delta$ is the front wheel angle; $\beta$ is side slip angle; $\alpha_{1}$ is the front wheel slip angle; $\alpha_{2}$ is the rear wheel slip angle; $m$ is the total weight; $v_{1}$ is velocity at center of mass; $v$ is $v_{l}$ component at time $t$ on oy axis; $u$ is $v_{l}$ component at time $t$ on ox axis; $F_{y 1}, F_{y 2}$ are respectively cornering force of the ground on the front and rear wheel; $\zeta$ is the angle between the front wheel speed direction and the ox axis; $O$ is the origin of the coordinate axis; $C$ is the center of mass of vehicle; $O^{\prime}$ is the instantaneous steering center.

\subsection{Establish differential equation of vehicle dynamics}

According to the above-mentioned modeling analysis, the differential equation of vehicle motion at front wheel angle step input can be deduced as follows:

$$
\left\{\begin{array}{l}
\left(k_{1}+k_{2}\right) \beta+\frac{1}{u}\left(a k_{1}-b k_{2}\right) \omega_{r}-k_{1} \delta=m\left(\dot{v}+u \omega_{r}\right) \\
\left(a k_{1}-b k_{2}\right) \beta+\frac{1}{u}\left(a^{2} k_{1}+b^{2} k_{2}\right) \omega_{r}-a k_{1} \delta=I_{z} \omega_{r}
\end{array}\right.
$$




\subsection{Two-DOF vehicle model Based on Simulink}

According to formula (1), Two-DOF vehicle simulation model established in Matlab / Simulink software environment is shown in Figure.2. The assignment of each parameter is shown in Table 1.

Table 1. Two-DOF vehicle model simulation parameters

\begin{tabular}{|c|c|}
\hline Model parameter & Value \\
\hline Mass $\mathrm{m}(\mathrm{Kg})$ & 1000 \\
\hline Rotational inertia $\mathrm{Iz}\left(\mathrm{Kgm}^{2}\right)$ & 2800 \\
\hline Distance from centroid to front axle a $(\mathrm{m})$ & 1.3 \\
\hline Distance from centroid to rear axle $\mathrm{b}(\mathrm{m})$ & 1.2 \\
\hline Front wheel cornering stiffness k1 $(\mathrm{N} / \mathrm{rad})$ & 51000 \\
\hline Rear wheel cornering stiffness $\mathrm{k} 2(\mathrm{~N} / \mathrm{rad})$ & 45000 \\
\hline
\end{tabular}

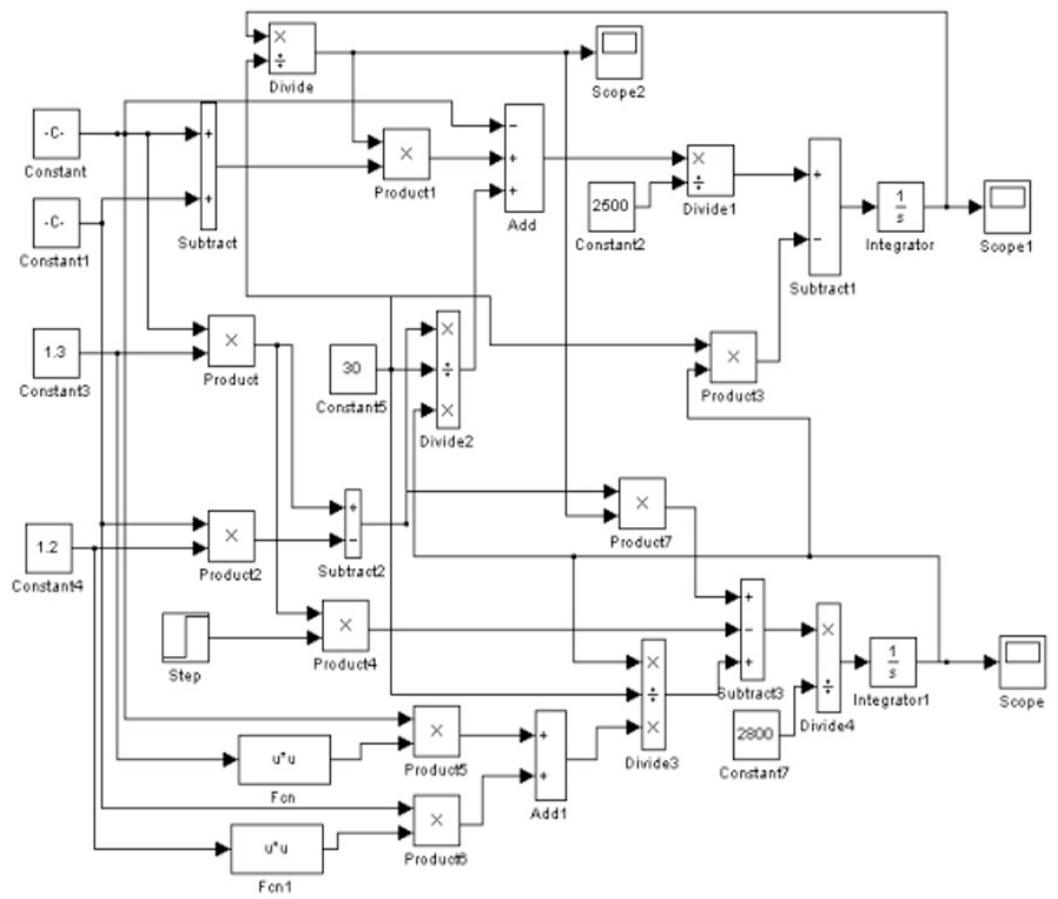

Figure 2. Simulation model of two-DOF vehicle.

\section{Simulation analysis}

According to the established simulation model, we set the front wheel turning angle input as the angular step signal, the amplitude is $0.4 \mathrm{rad}$, and the simulation time is $5 \mathrm{~s}$. The response curve of the yaw velocity and side slip angle at three different vehicle speeds are obtained, as shown in Figure.3, 4. 


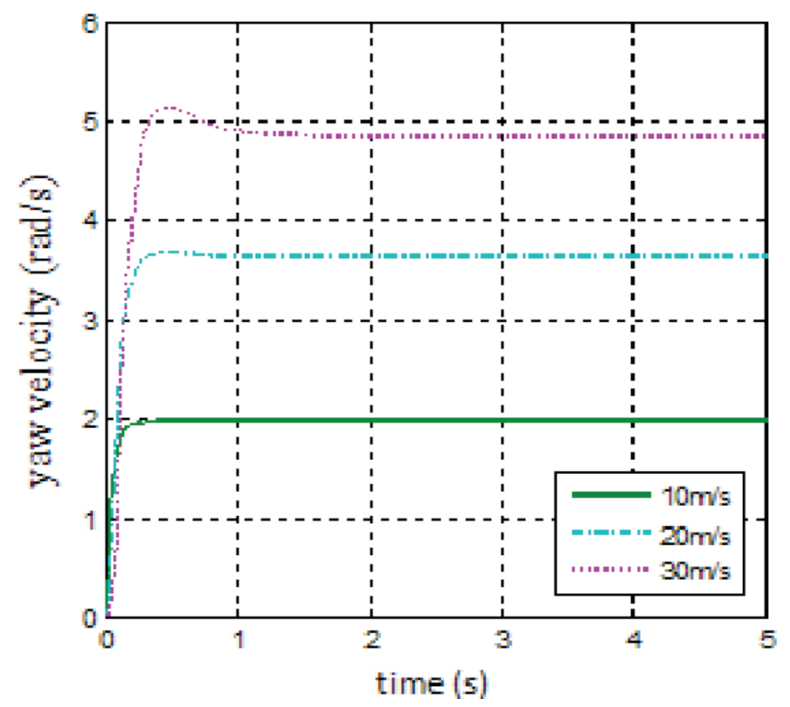

Figure 3. Yaw velocity curve.

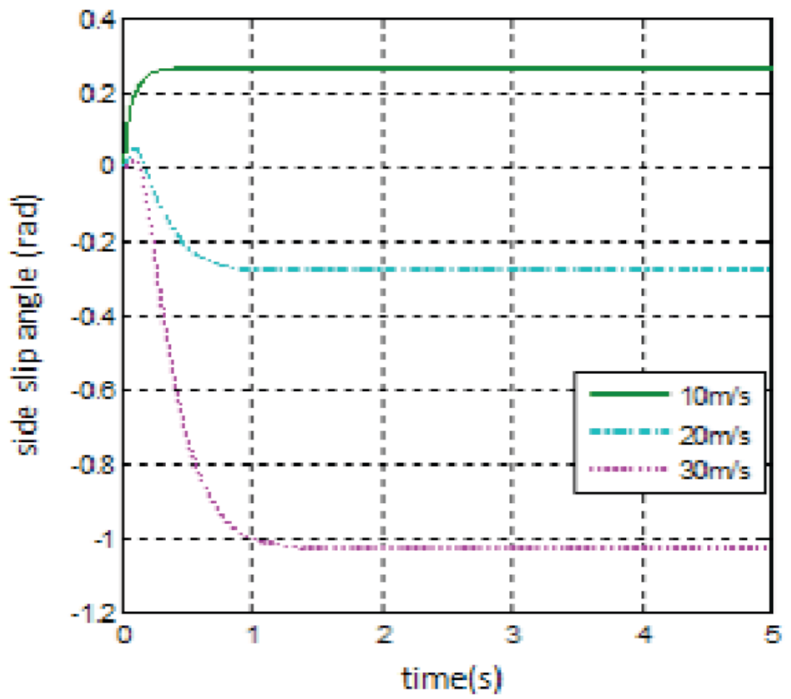

Figure 4. Curve of side slip angle.

It can be seen from Figure. 3 that, after a given angular step input, the vehicle yaw velocity response curve changes with the speed change. When the vehicle speed is $10 \mathrm{~m} / \mathrm{s}$, the yaw rate reaches stable at about $0.3 \mathrm{~s}$ after slight fluctuation and the steady state value is about $2 \mathrm{rad} / \mathrm{s}$, and the steady state response time is short. It can be seen from Figure.4 that when the vehicle speed is $10 \mathrm{~m} / \mathrm{s}$, the vehicle side slip angle is stable at about $0.3 \mathrm{~s}$, and the steady state value is about $0.28 \mathrm{rad}$. When the vehicle speed is $30 \mathrm{~m} / \mathrm{s}$, the yaw rate reaches stable at about $1 \mathrm{~s}$, and the steady state value is about 4.8 $\mathrm{rad} / \mathrm{s}$, the yaw velocity fluctuates more at high speed than low speed, and the steady-state response time becomes longer; The side slip angle is stable at $1.3 \mathrm{~s}$, and the steady state value is about $-1.01 \mathrm{rad}$, which is much higher than the value when the vehicle speed is $10 \mathrm{~m} / \mathrm{s}$. 
From the above analysis, we can see that when driving at a lower speed, vehicle enjoys better transient response characteristics. With the increase of vehicle speed, the response amplitude of yaw velocity and side slip angle becomes larger and the stabilization time becomes longer. This rule is in good agreement with the actual situation, which verifies correctness of the established model. Subsequently, the model can be used for vehicle handling stability control algorithm and experimental research.

\section{Conclusion}

Vehicle handling stability has a direct impact on steering characteristics, power output characteristics, driving safety, etc., so it is necessary to carry out further research on it. Based on the theory of vehicle handling stability, a simulation model of linear two-DOF vehicle handling stability is established. The model can be used to analyze the yaw velocity and side slip angle response at front wheel corner step input at any given vehicle speed. Through analysis of the simulation results, it can be seen that the response cure of vehicle yaw velocity and side slip angle in the time domain simulated by the model can more intuitively reflect change of vehicle handling stability, which has a certain reference value for the future experimental study.

\section{Acknowledgement}

The authors would like to thank Anhui province science and technology research key project (Grant No. 1604a0902158) and Anhui university scientific research platform innovation team building projects (2016-2018).

\section{References}

1. J. Liu. Dynamics Model and Simulation Analysis of Half-car Suspension Based on MATLAB/Simulink, J. Journal of Jiamusi University(Natural Science Edition), (06): 948-951. (2015)

2. J. Li, S.H. Yuan, D.M. Ju, R.Y. Li. Research on Test Methods for Special Vehicle Handling and Stability Based on Dynamics Simulation, J. Acta Armamentarii, (02): 262-267. (2014)

3. W. Huang, X.H. Wang, M. Zhang. Simulation on Handling Stability of Mult-iaxle Steering Vehicle, J. Tractor \& Farm Transporter, (01): 70-73+81. (2011)

4. F.B. Bao. Analysis based on Vehicle Handling Stability, J. Western China Communications Science \& Technology, (11):75-79. (2011)

5. Q.D. Wang, G.H. Zhang, W.W. Chen, H. Zhu. A Study of Vehicle Dynamics Stability Control Based on Variable Structure Control, J. China Mechanical Engineering, (05): 622-626. ( 2009)

6. H. Wang, L. Yang, S.H. He, C.W. Peng. Co-simulating the Vehicle Controlling Stability Based on ADAMS and MATLAB, J. Agricultural Equipment \& Vehicle Engineering, (08): 38-41. (2012)

7. T.J. Chen, Y. Chen, F. Zhao, Q.L. Zhou. Control and Simulation Analysis of Handling and Stability of Electric Vehicle with All Motorized Wheels, J. Journal of System Simulation, (12): 3033-3038. (2013)

8. S. Chen, S. Meng, G. Li, Y.Y. Qin, X.Z. Qu. The Study on Co-Simulation Modeling of Automobile Ride and Handling, J. Agricultural Equipment \& Vehicle Engineering, (04): 12-16. (2016)

9. X.W. Han, F. He, Y.D. Zhang, J.S. Chen. Roll Stability Analysis of Heavy Vehicles Based on Fuzzy Control, J. Coal Mine Machinery, (04): 7-9. (2016)

10. W.P. Li, S.J. Mo, J. Xiao, B.Z. Zhang. Control and Optimization of Vehicle Roll Stability with Considering of Ride Comfort, J. Computer Simulation, (08): 180-184. ( 2015) 
11. S.B. Zhang, M. Li, Y.X. Liu. Simulation Analysis and Influencing Factor Study of Vehicle Handling Stability, J. Tractor \& Farm Transporter, (02): 23-26+34. (2015) 